\title{
1 Individual Learning Phenotypes Drive Collective Cognition
}

3 Chelsea N. Cook ${ }^{1 *}$, Natalie J. Lemanski², Thiago Mosqueiro², Cahit Ozturk ${ }^{1}$, Jürgen

4 Gadau $^{3}$, Noa Pinter-Wollman ${ }^{2 \diamond}$, Brian H. Smith ${ }^{1 \diamond}$

\section{Affiliations}

$7 \quad{ }^{1}$ School of Life Sciences, Arizona State University, Tempe, USA

$8{ }^{2}$ Department of Ecology and Evolutionary Biology, University of California Los Angeles,

9 Los Angeles, USA

$10{ }^{3}$ Institute for Evolution and Biodiversity, University of Münster, Münster, Germany

$11{ }^{\circ}$ Co-Senior Authors

12 'Corresponding Author, cncook1@asu.edu

Classification: Major: Evolution, Minor: Ecology

Keywords: Collective Cognition, Collective Behavior, Learning, Latent Inhibition, Honey Bees, Foraging

Author Contributions: CNC, TM, JG, NPW, BHS conceived of and helped design the study. CNC, NJL, and TM analyzed the data. CNC and CO created the genetically selected lines and CO maintained them. CNC carried out data collection and wrote the first draft of the manuscript. CNC, TM, NJL, NPW, BHS discussed results. All authors commented on the manuscript.

This PDF includes:

26 Main Text

27 Figures 1-4

28 Supplemental Materials

29 Figure S1

30 Tables S1 to S8 


\section{Abstract}

33 Variation in cognition can influence how individuals respond to and communicate about

34 their environment, which may scale to shape how a collective solves a cognitive task.

35 However, few empirical examples of variation in collective cognition emerges from

36 variation in individual cognition exist. Here, we show that interactions among individuals

37 that differ in the performance of a cognitive task drives collective foraging behavior in

38 honey bee colonies by utilizing a naturally variable and heritable learning behavior

39 called latent inhibition (LI). We artificially selected two distinct phenotypes: high LI bees

40 that are better at ignoring previously unrewarding familiar stimuli, and low LI bees that

41 can learn previously unrewarding and novel stimuli equally well. We then provided

42 colonies composed of these distinct phenotypes with a choice between a familiar feeder

43 or a novel feeder. Colonies of high LI individuals preferred to visit familiar food locations,

44 while low LI colonies visited novel and familiar food locations equally. However, in

45 colonies of mixed learning phenotypes, the low LI bees showed a preference to visiting

46 familiar feeders, which contrasts with their behavior when in a uniform low LI group. We

47 show that the shift in feeder preference of low LI bees is driven by foragers of the high

48 LI phenotype dancing more intensely and attracting more followers. Our results reveal

49 that cognitive abilities of individuals and their interactions drive emergent collective

50 outcomes.

\section{Significance Statement:}

53 Variation in individual cognition affects how animals perceive their environment and which

54 information they share with others. Here we provide empirical evidence that how individual

55 honey bees learn contributes to collective cognition of a colony. By creating colonies of distinct

56 learning phenotypes, we evaluated how bees make foraging choices in the field. Colonies

57 containing individuals that learn to ignore unimportant information preferred familiar food

58 locations, however colonies of individuals that are unable to ignore familiar information visit

59 novel and familiar feeders equally. A 50/50 mix of these phenotypes prefer familiar food

60 locations, because individuals who learn the familiar location recruit nestmates by dancing more

61 intensely. Our results reveal that variation in individual cognition scales non-linearly to shape

62 collective outcomes. 


\section{INTRODUCTION}

Collective behavior allows animals to undertake tasks that they could not accomplish alone. Individuals utilize local information to adjust to ecological changes as a collective. Local information is implicitly or explicitly communicated among group members to form a collective response (1-3). Individuals within a group vary in their cognitive abilities. Cognition at the individual level occurs when an organism perceives, integrates, and utilizes acquired information. Collective cognition is a form of collective behavior that emerges from the interactions among individuals working together to solve a cognitive task that could not be accomplished as effectively at the individual level (1, 4). Many of the basic rules that explain collective behavior and cognition come from theoretical modes, which emphasize the importance of variation in perception and cognition among individuals within a social group (5). For example, leaders can emerge in computer simulations to guide uninformed group members to a resource. However, both informed and uninformed individuals are needed to effectively move in the correct direction (6). Although individual variation in responsiveness and cognitive ability is recognized as critical for the emergence of collective cognition, empirical work on the mechanisms by which variation in individual cognition and the interaction between these different behavioral types scales to the collective are rare.

One way in which animals differ from one another in their cognitive abilities is the way in which they perceive information (7). This perception may be driven by several cognitive properties, including the ability to learn relevant information. This ability has important ecological and evolutionary consequences(8). For example, learning is the foundation of the evolution of aposematic coloration (9). Humans that are able to quickly learn important information report increased productivity compared with individuals that cannot focus on pertinent information (10-12). Naturally, collective groups of organisms will consist of individuals that vary in how they learn information. Here we ask how

91 individual variation in learning shapes the way in which individuals learn and share

92 ecological information with group members to shape collective outcomes. 
While foraging, honey bees (Apis meillifera) must learn various aspects about the location of food sources, such as landmarks, odors, and direction (13-15). Honey bee foragers then return to the colony to communicate this spatial information to colony members at the nest via their recruitment dances(13). In the lab, honey bees exhibit variation in their ability to learn to ignore unimportant information, such as unrewarding odors, known as latent inhibition $(16,17)$. LI has been studied in vertebrates (18-22) and is correlated with attention disorders in humans (10). $\mathrm{LI}$ is heritable in honey bees (23). Foraging honey bees vary in their expression of $\mathrm{LI}$; scouts tend to exhibit high LI and ignore familiar odors, while recruits tend to exhibit low LI and learn familiar and novel odors equally well (24). Despite our knowledge of variation among individuals in latent inhibition $(23,25)$, and its effects on predator avoidance $(18,19,26)$, it is unknown whether or how this variation affects ecologically relevant decisions in social systems.

We provide empirical evidence that the interaction of individuals that vary in their cognitive abilities drives collective cognition. Using the genetic heritability of LI, we first tested reproductive queen and drone honey bees to characterize their LI, then we selected two distinct phenotypes from the reproductive individuals: high LI and low LI.

We then created genetic learning lines from singly inseminated queens by like

113 performing drones to produce two distinct lines of workers that exhibit similar LI to their 114 parents. First, we verify that the social environment of adult honey bees from selected 115 lines does not affect their LI phenotypes as foragers. We then created 24 colonies 116 composed of single cohorts of only low, only high, 50/50 mixed high and low LI workers, 117 as well as age-matched non-selected control bees. To compare collective foraging 118 behavior across these selected colonies, we placed them in semi-natural foraging 119 conditions, then evaluated the number of forager visits, first visits, and re-visits to the 120 familiar or novel food locations. To explore the mechanisms underlying how individual 121 variation in LI affects collective foraging, we quantified the round recruitment dance in 6 122 mixed colonies while the colonies visited novel and familiar feeders. These experiments 123 allowed us to simultaneously quantify how collectives vary in performing cognitive tasks 
124 as a result of the composition of the individuals of that collective, as well as how

125 cognitively distinct individuals interact to shape collective outcomes.

\section{RESULTS}

To ensure workers in different social environments exhibited the predicted

130 heritable LI phenotype, we evaluated the LI score of foragers after 21 days in either

131 their natal colony or a control colony. We marked 1000 individuals from each selected

132 line (high or low LI) on the day of emergence. We then placed 500 individuals back into

133 their natal colony and 500 individuals into an established control colony of equal size

134 with an open mated queen, i.e. workers with a variety of learning phenotypes. We

135 monitored the colonies until marked bees began to make foraging flights ( 21 days). We

136 then collected marked foragers as they returned to the colony and brought them into the

137 laboratory to evaluate their LI. We avoided pollen foragers as they tend to exhibit

138 different learning behavior compared to nectar foragers(27). We found that foragers

139 retained the expected LI based on the LI of their parents, regardless of whether they

140 were housed with same or with variable learning phenotypes. Foragers from the high

141 and low lines differed in expression of $L I$ as expected (GLM: $\chi^{2}=4.84, d f=1, p=0.027$,

142 Figure 1). We did not detect an effect of the identity of the colony in which the bees

143 were housed on LI phenotype $\left(\chi^{2}=3.28, d f=2, p=0.193\right.$, Figure 1). 


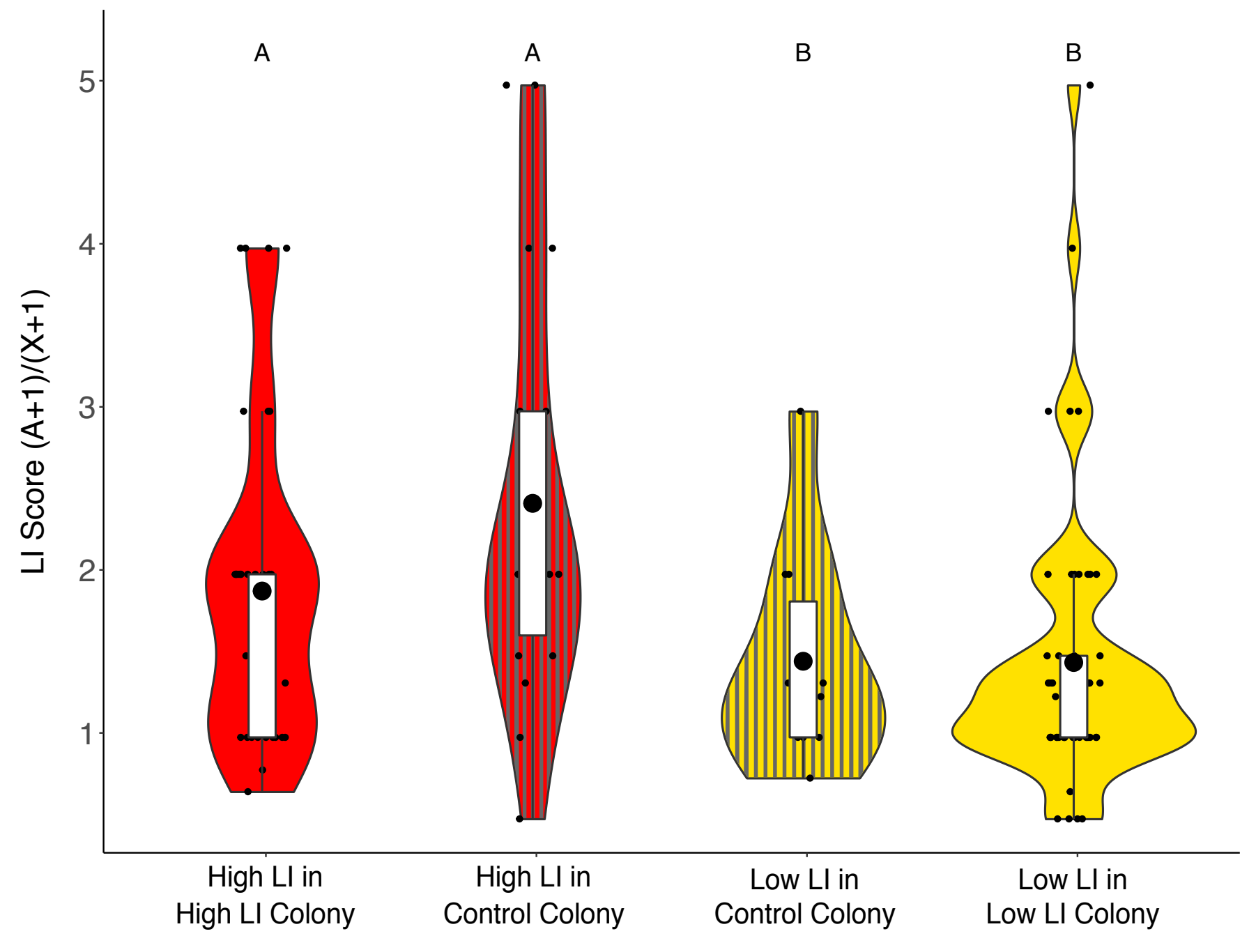
latent inhibition. LI scores of individuals from high $\mathrm{LI}$ lines that spent their adult life either in high LI only colonies (red, $\mathrm{n}=36$ ) or in a control colony with a variety of LI phenotypes from an open mated queen (red with gray vertical lines, $n=18$ ); individuals from low LI lines that spent their adult life either in low LI only colonies (yellow, $n=52$ ) or in control colonies (yellow with gray vertical lines, $n=10$ ). In this and subsequent figures, the large black dot is the mean, the white box is the interquartile range (IQR), whiskers extend to $1.5^{\star} \mathrm{IQR}$, and the small points beyond the whiskers are outliers. Shaded areas show the distribution of the data. Here, and in all following figures, yellow are low LI colonies and individuals, gray are control colonies and individuals, and red are high LI colonies and individuals. Attention is critical for many individual behaviors, including finding the correct mate or prey. 
To determine how the learning phenotypes influenced colony-level foraging behavior, we placed small single-cohort (same age bees) colonies into a flight cage and monitored foraging activity. We evaluated 4 colony types each week: one control colony consisting of approximately 1300 age-matched bees from open mated queens; one colony consisting of 650 workers from high LI queens plus 650 age-matched control bees; one colony consisting of 650 workers from low LI queens plus 650 aged-matched control bees; and one 50/50 mixed colony with 325 workers from each LI line plus 650 aged-matched control bees. In the last 3 types, the supplemented 650 age-matched bees from open mated queens were used to ensure a small but functioning colony as we did not have enough workers from the single-drone-inseminated queens and colonies of just 650 individuals would be too weak to forage. Honey bee division of labor is largely influenced by worker age, so we used age-matched bees to remove any

174 influence that age may have on foraging propensity. On day 1, we trained bees to a 175 feeder inside the tent containing $1 \mathrm{M}$ sucrose and an odor, which became the 'familiar' feeder. During the subsequent 3 days, in addition to the familiar feeder, we introduced a single novel feeder each day with a different odor and color, but with the same sugar concentration as the familiar feeder (Figure 2A). To evaluate the collective ability of the colony to find a new feeder, we recorded the number of visits to each feeder by bees from each selected line according to the color of paint on the bees' thorax. We further marked bees with a feeder-specific color on their abdomen when they visited the feeder for the first time to determine if bees revisited that feeder. We repeated this for 6 weeks on 6 colonies for each group type.

Colony composition strongly influenced overall number of visits to the food locations $\left(N=6\right.$ colonies in each line, 24 total, 6172 total visits; $G L M: \chi^{2}=1270$, df $=3$,

187 compared to low LI colonies (Tukey post hoc: $Z=25.5, p<0.0001$, Figure 2A), mixed colonies $(Z=5.18, p<0.0001)$, and controls $(Z=26.6, p<0.0001)$. Mixed $\mathrm{LI}$ colonies also had significantly more visits compared to low $(Z=20.7, p<0.0001)$ and controls $(Z=21.8$,

$190 \mathrm{p}<0.0001)$. Low LI and control colonies had the fewest total visits and were not

191 significantly different from each other $(Z=-1.38, p=0.50)$. 
Foraging in the high, low, and control colonies was largely performed by bees revisiting the feeders. (GLM, $\chi^{2}=22.32$, df $=3, p<0.0001$, Figure $2 \mathrm{C}$ ). However, the mixed LI colonies had a significantly lower proportion of revisiting foragers compared to 195 the low (Tukey post hoc: $Z=-4.2, p=0.0002)$, high $(Z=-3.1, p=0.01)$, and control colonies $196(Z=-3.33, p=0.004)$. We did not detect significant differences among the other colony 197 types (See Supplementary Table 3).

A colony's LI phenotype composition determined its preference between the novel and familiar feeders (GLM: Feeder ${ }^{*}$ Colony $\chi^{2}=473.64, \mathrm{df}=3, \mathrm{p}<0.0001$; Figure

200 2D). High and mixed colonies preferred the familiar feeder over the novel one (Tukey

201 Posthoc: High Familiar:Novel: Z=20.2, p<0.0001; Mixed Familiar:Novel: Z=25.6,

$202 \mathrm{p}<0.0001)$. Low LI and control colonies did not show a strong preference for either

203 feeder, visiting them equally (Low Familiar:Novel: Z=-1.24, $p=0.92$; Control

204 Familiar:Novel: $Z=2.03, p=0.46)$. For full pairwise comparisons, see Supplementary

205 Table 4).

206 The number of re-visits to the novel and familiar feeders was different across 207 colony compositions (Figure 2E: Colony*Feeder $\chi^{2}=53.67, p<0.0001$ ). All colonies had

208 a higher proportion of re-visits to the familiar feeder compared to the novel feeder.

209 However, the mixed LI colonies had a much lower proportion of re-visitation to the novel 210 feeders than the other colony types (Supplementary Table 5). Thus, new foragers in the 211 mixed colonies that visited the novel feeder were less likely to return to it compared to 212 foragers who visited the novel feeders in other colonies. 

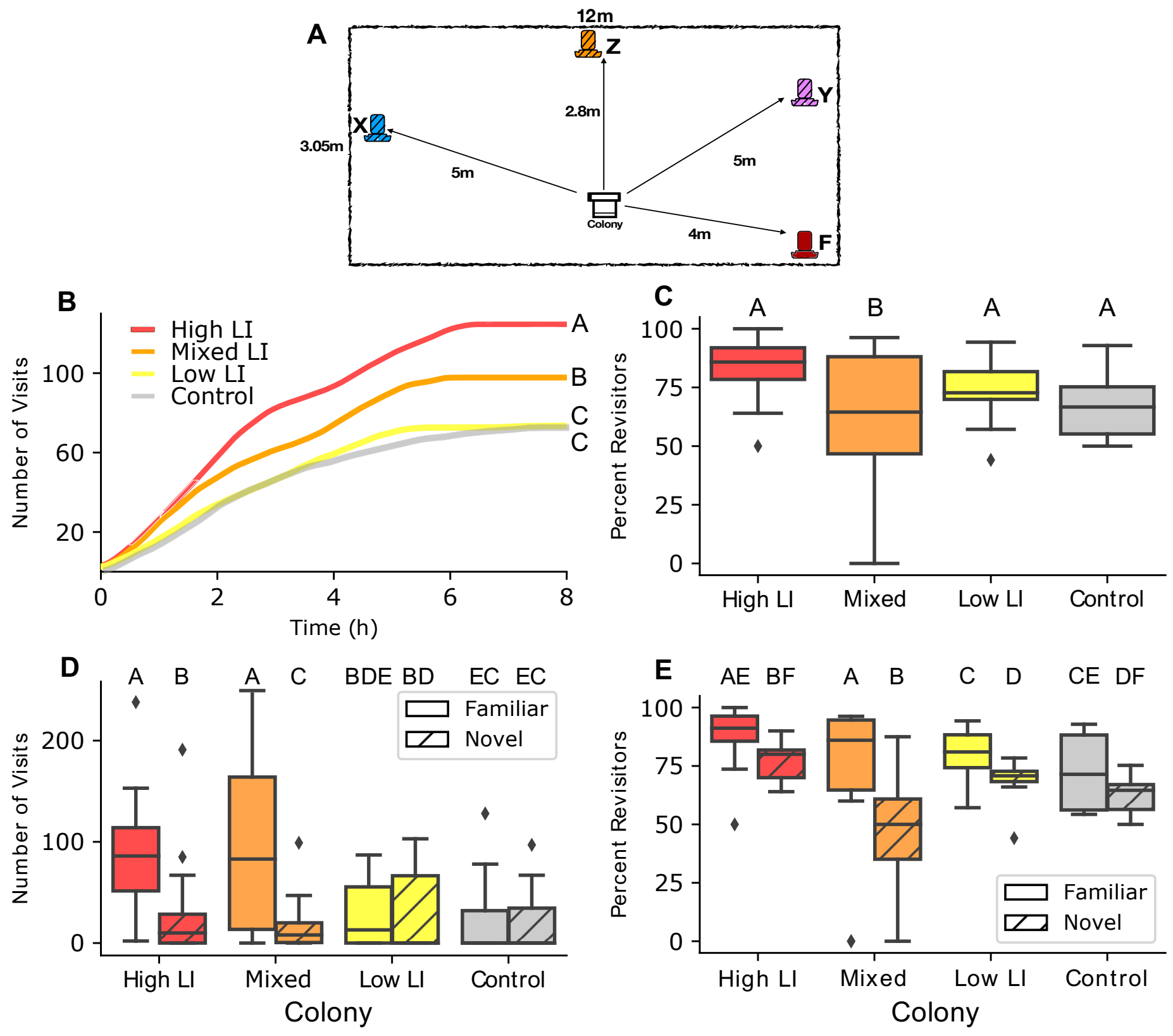

Figure 2: Colonies constructed from different genetic lines selected for high or experimental set up illustrating the location of feeders in relation to the location of the colony (center, white) within the experimental arena (large rectangle). The familiar feeder (red) was provided on day 1 and on all subsequent days. Novel feeder $X$ (blue) was presented on day 2 , novel feeder $Y$ (purple) on day 3 , and novel feeder $Z$ (orange) on day 4. See Supplementary Table 2 for associated odors. Visits to all novel feeders were combined for statistical analysis. (B) Cumulative number of visits of bees to all feeders over time by colony type. Different letters to the right of the lines indicate 
illustration of visitation by each colony on each day, see Supplementary Figure 1. (C) Percent of re-visits out of the total number of visits to all feeders by colony type. Here and in all following panels, different letters above boxes indicate statistically significant differences according to a post hoc Tukey test. (D) Number of all visits to the familiar feeder (solid boxes) and a novel feeder (hatched boxes) for each type of colony, when both novel and familiar feeders were presented simultaneously (days 2-4). (E) Percent of re-visits out of the total number of visits to either the familiar or the novel feeder by type of colony when both novel and familiar feeders were presented simultaneously (days 2-4). In C, D and E, horizontal lines are the median, the boxes are the interquartile range (IQR), whiskers extend to $1.5^{*} \mathrm{IQR}$, and the small points beyond the whiskers are outliers. $\mathrm{N}=24$ colonies, 6 colonies per group type, 6172 total visits.

To determine why the mixed colonies showed a preference for the familiar feeder (Figure 2D), we examined how individual lines visited each feeder (Figure 3). In 2017, we tested mixed colonies placed in a flight cage. In 2018, we reselected lines and then placed mixed colonies into two-frame observation hives to evaluate recruitment dances along with visitation to the feeders in the flight cages. We found that there was a significant year effect (Supplementary Table 6), likely due to reselection and different environmental conditions. We therefore statistically analyzed each year separately to focus on the within-year variation between the selected lines. mixed with high LI bees. In 2017, we found a significant interaction between the selected line and which feeder foragers visited (GLM: $\chi^{2}=7.79, \mathrm{df}=2, \mathrm{p}=0.02$; Figure

249 familiar feeder when they had a uniform colony composition (Figure 2E), when mixed 250 with high LI individuals, low LI and control individuals exhibited a preference to the 251 familiar feeder (GLM: Low Familiar:Novel: Z=13.28, $p<0.0001$; Control Familiar:Novel: 252 Z=18.32, p<0.0001; Figure 3A). High LI individuals showed a preference to familiar 253 feeders (GLM: Familiar:Novel: Z=22.03, p<0.0001) just as colonies comprised of only 254 high LI individuals did (Figure 2E). We found a significant interaction between selected 255 line and feeder in 2018 (GLM: $\chi^{2}=85.27, \mathrm{df}=2, \mathrm{p}<0.0001$; Figure 3B), with low LI and 256 control individuals showing preference to the familiar feeder over the novel feeder 257 (GLM: Low Familiar:Novel: Z=25.05, p<0.0001; Control Familiar:Novel: Z=13.90, $258 \quad \mathrm{p}<0.0001$; Figure 3B) similar to high LI individuals preferring the familiar feeders 
259 (Familiar:Novel: $Z=18.48, p<0.0001)$. For full contrasts from 2017 see Supplementary

260 Table 7, for 2018 see Supplementary Table 8.
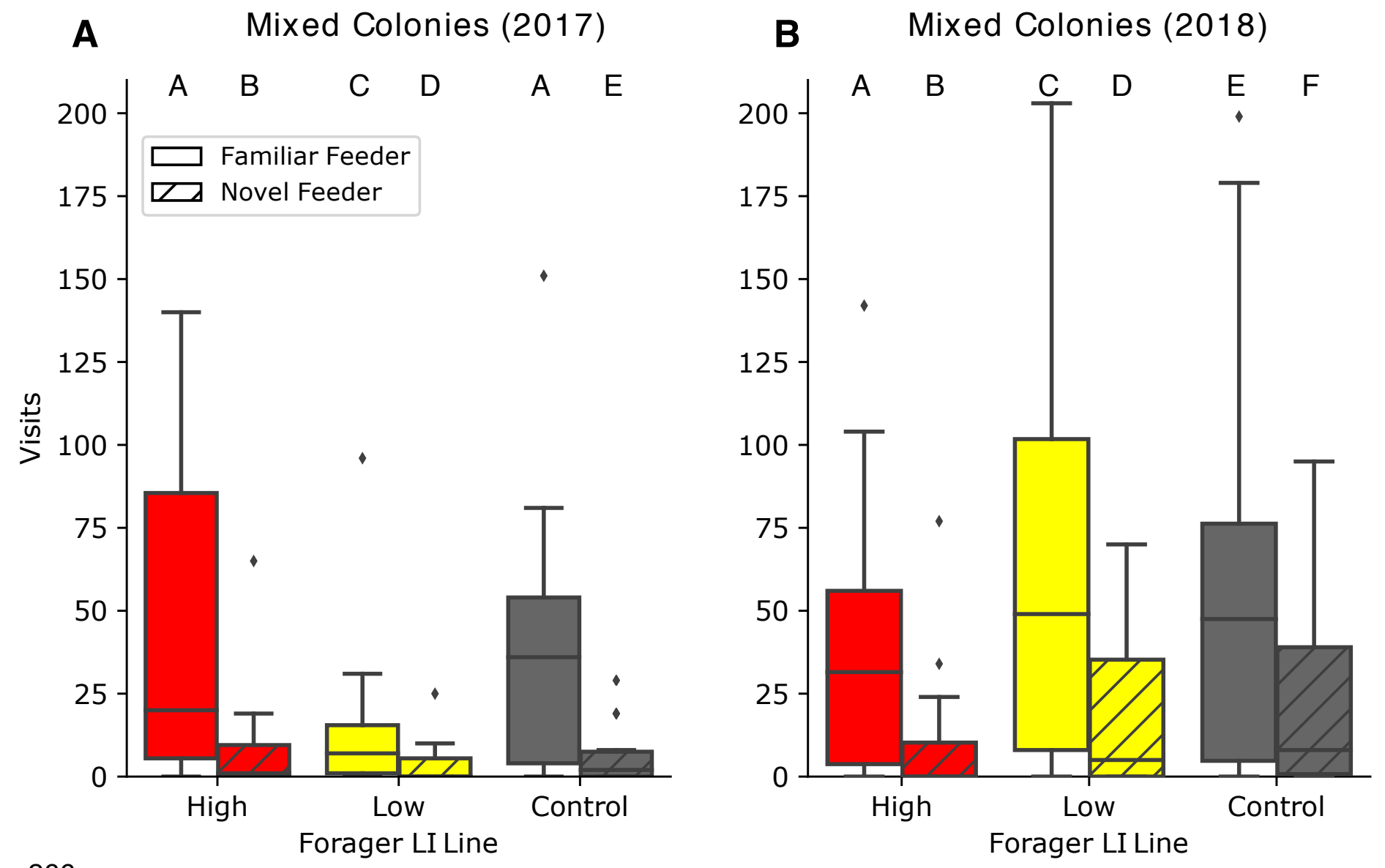
mixed colony. Daily visits to the familiar (solid) and novel (hatched) feeders by individual bees in mixed colonies from low LI parents (yellow), high LI parents (red) or open mated queens (grey) in (A) 2017, N=6 mixed colonies, 2347 overall visits and (B) in mixed colonies from lines that were re-selected in $2018, N=6$ colonies, 6272 overall visits. The horizontal line in the box is the median, the box is $25-75 \%$ of the data, whiskers represent $95 \%$ of the data, and diamonds show outliers beyond $95 \%$. Different letters above boxes indicate statistically significant differences according to a post hoc Tukey test. 
To uncover the behavioral mechanisms that underlie the switching of low LI individuals from having no feeder preference when in a uniform colony composition to preferring the familiar feeder when in a mixed colony, we examined the round dance,

283 the modified waggle dance used at short distances(28), of individuals from each selected line in mixed colonies as they returned from foraging. Using observation hives with glass walls, we video recorded bees performing the round dance to recruit other individuals in the colony to forage. To determine which selected line recruited to each feeder, we noted the selected line of the dancer (high or low LI) according to the paint marks on the individuals' thorax and whether the dancer had visited a feeder according to the paint marks on abdomens. We did not record dancers without abdominal marks as they were likely collecting from and recruiting to unmonitored water sources. To determine who the information about a feeder was communicated to, we counted the number of followers of each dancer and the selected line of the followers. To quantify the dance intensity, we recorded the duration of the dance, and the number of turns the dancer made during the first 20 seconds of the dance.

Individuals from the lines differed in their likelihood to perform a round dance (Chi-square test: $\chi^{2}=26.61$, df=2, $p<0.0001$; Figure 4B). Low LI individuals were significantly more likely to perform a dance compared to high Li individuals (pairwise chi-square test: $p=0.0001$ ) and controls (pairwise chi-squared test: $p=0.004$ ). High LI individuals were just as likely to perform a dance as controls $(p=0.36)$. Individuals differed in their likelihood to follow a dance based on their selected line (Chi-square test: $\chi^{2}=28.26, \mathrm{df}=2, \mathrm{p}<0.0001$; Figure 4B). Low LI individuals were significantly more likely to follow a dance compared to high LI bees (pairwise chi-square test: $p<0.0001$ ) and controls (pairwise chi-square test: $\mathrm{p}<0.003$ ). High LI and control individuals were equally likely to follow a dance (pairwise chi-square test: $p=0.240$ ).

Although the high LI individuals danced less often, high LI dances had significantly more followers compared to low and control bees (Chi-square test: $\chi^{2}=$ 307 13.93, df=2, $\mathrm{p}<0.001$; Figure 4C). Low LI bees performed more dances that had no 308 followers compared to high LI and control dances. We did not detect a statistically 309 significant difference between the proportion of individuals from each line that followed 
310 each line of dancer (Chi-square test: $\chi^{2}=7.05$, df $=4, p=0.13$, Figure 4D). Low LI

311 individuals spent more time dancing; however they attracted fewer followers than high

312 and control dancers, indicated by the significant interaction between the LI of the dancer

313 and dance duration when predicting the number of followers (GLMM: $\chi^{2}=6.42, \mathrm{df}=2$,

$314 p=0.04$; Figure 4E).

315 The relative attraction of dances of high LI bees could be due to the intensity of

316 the dance. High LI bees performed more turns per second during their dances (ANOVA:

$317 \chi^{2}=12.8, \mathrm{df}=2, \mathrm{p}=0.002$; Figure 4F). High LI dancers performed an average of 0.59 turns

318 per second, significantly higher than low LI dancers, who performed an average of 0.52

319 turns per second (Tukey: $t=-3.13, p=0.005)$. Control bees also performed more turns per

320 second than low LI bees (Tukey: $t=-2.5, p=0.03$ ), but not different than high LI bees, at

321 an average 0.62 turns per second (Tukey: $t=-0.7, p=0.7$ ). 

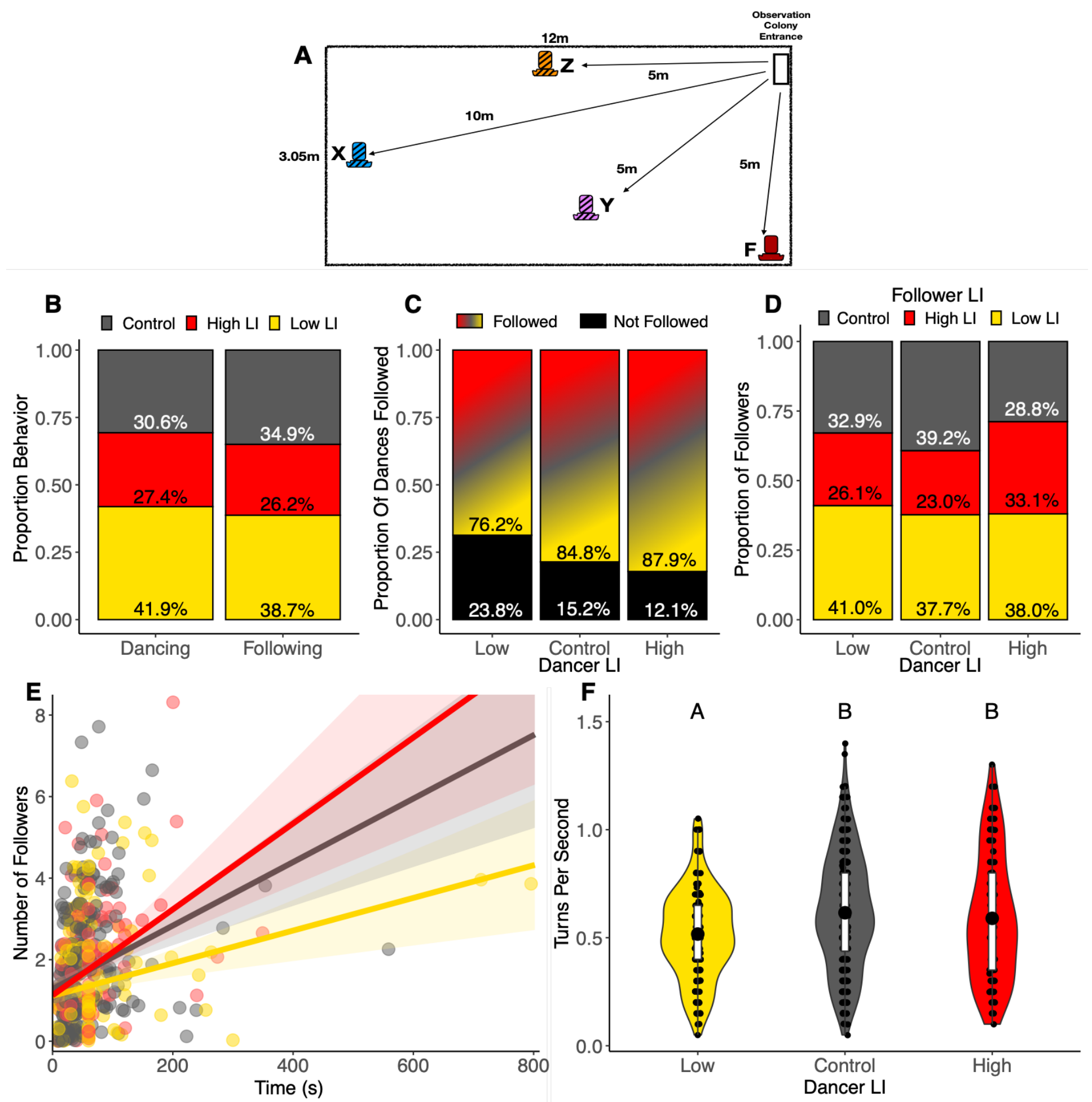

324 Figure 4: Recruitment dances facilitate integration of information from different

325 genetically selected lines. (A) The experimental set up illustrating the location of

326 feeders in relation to the location of the colony entrance (top right, white) within the

327 experimental arena (large rectangle). The familiar feeder (red) was provided on day 1 
and on all subsequent days. Novel feeder $X$ (blue) was presented on day 2, novel feeder $Y$ (purple) on day 3, and novel feeder $Z$ (orange) on day 4. See supplementary table 2 for associated odors. Visitation to novel feeders were combined for statistical analysis. (B) Proportion of dances $(N=667)$ or follows $(N=1201)$ across 6 colonies performed by bees from each line, relative to their abundance in the mixed colony (350 high, 350 low, 700 control). We accounted for the difference in abundance of each selected line by dividing the number of observed control dancers by 2 before calculating these proportions. (C) Proportion of dances performed per LI line type that were either followed by at least one individual (colored) or not followed by any other bees (black). (D) Proportion of dances by LI line type that were followed (from panel B) broken down by LI of the follower. (E) Relationship between number of followers and duration of a dance by line. Point and line colors indicate $\mathrm{LI}$ of dancer. Best fit line represents the GLM, shaded area represents the 95\% confidence interval. (F) Rate of turns per second in a dance by line. The large black dot in the box is the mean, the box is $25-75 \%$ of the data, whiskers represent $95 \%$ of the data. The violin shapes illustrate distribution of the data. Different letters above violins indicate statistically significant differences according to a post hoc Tukey test.

\section{DISCUSSION}

By combining techniques from experimental psychology and behavioral ecology, we have developed a system for investigating how variation in individual learning behavior drives collective cognition. We utilized this system to demonstrate that a laboratory-selected heritable learning behavior with natural individual variation scales to shape the collective performance of a honey bee colony on foraging tasks. In the lab, high LI honey bees learn to ignore familiar odors that they experienced without reinforcement, while readily learning novel odors. When a stimulus is rewarding, high LI bees exhibit increased attention to that information. One interpretation of reduced learning to a familiar, unrewarding, stimulus is that pre-exposure reduces attention to, and thus associability of, that stimulus. This interpretation is an extension of conditioned attention theory $(29,30)$, which proposes that latent inhibition is induced by allowing animals to focus their attention on important information(30-32). Our observations of

361 interpretation, whereby high LI individuals have stronger attention capacities to food compared to low LI individuals. Once high LI individuals have found a food location, 
363 they continue to revisit it, 'attending' more strongly to reinforced feeders over new ones.

364 The increased impact of the resource on these bees could translate into stronger, more

365 vigorous dances. In contrast, low LI individuals learn and visit both known and new

366 feeders equally, dividing their attention across resources and acting more like generalist

367 foragers. In mixed colonies, this broadened attention by low LI individuals may therefore

368 make them the perfect audience for the high LI dancers, driving them to prefer feeders

369 that high LI individuals preferentially visit. Under natural conditions, where queens mate

370 with many different drones, most colonies would possess both types of learners,

371 perhaps more closely resembling our mixed colonies(33). Attention is critical for many

372 individual behaviors, including finding the correct mate or prey(34). We propose that this

373 diversity of 'attention' aspect of individual cognitive phenotypes may enhance the overall

374 efficacy with which a group finds and exploits resources(35). In summary, our work

375 indicates that individual cognition scales to shape the collective cognition of animals

376 solving critical ecologically relevant tasks. 


\section{MATERIALS AND METHODS}

\section{Obtaining queens and drones}

To obtain queens for producing selected lines of a specific LI behavior, we performed the $\mathrm{LI}$ assay as outlined in $(23,24)$ on mature virgin queens 10 days after emergence. Briefly, we familiarized bees to an odor by puffing it at them 40 times every 5 minutes, then used the proboscis extension reflex to test their ability to learn to associate a food reward to the familiar versus a novel odor. Tested queens were placed into individually labelled queen cages and returned to a queenless colony until insemination, which typically occurred within a week of testing. To obtain fertile drones, we collected them from their returning unsuccessful mating flights at the entrance of colonies. We placed them into cages overnight in a queenless colony for $\mathrm{LI}$ testing the next day. After testing, drones were marked for individual identification and placed into a cage and placed into a queen bank for no longer than 3 days until inseminations occurred.

\section{Queen Inseminations}

We used instrumental insemination to inseminate a queen with sperm from a single drone. We inseminated a high LI queen with a high LI drone, and a low LI queen with a low LI drone $(36,37)$. LI varies across individuals. However, for this behavioral selection, we used the highest and lowest LI scoring individuals to create the high and low colonies, respectively. We then introduced queens to small queenless colonies, then allowed the queens to produce workers for 1 month. Colonies were checked

Cohoused Worker Preparation and Testing weekly to eliminate the possibility of supersedure.

To test the $\mathrm{LI}$ of foragers from each $\mathrm{LI}$ line, we placed frames of capped pupae from 3 high and 3 low LI colonies into $34^{\circ} \mathrm{C}$ incubators for 18 hours. After 18 hours, we used water based acrylic paint pens (Montana brand) to mark the abdomens of the

407 returned to their natal colony and half were placed into an established control colony of 
many are recognized as non-nestmates and rejected. After 2 weeks, colonies were monitored every day until marked bees began to forage, $\sim 21$ days after emergence. Returning nectar foragers were collected and tested for LI.

Field Colony Experimental Setup

To explore the colony-level foraging behavior of the LI lines, we set up 4 treatment colonies for each of the colony types: a high LI colony, a low LI colony, a $41650 / 50$ mixed colony, and a control. We ran weekly field experiments for 6 weeks. For 417 ease of identification, we always marked individuals from high LI colonies red, orange, 418 and pink, and individuals from low LI colonies green, blue, yellow, and white. We 419 continued to mark emerging bees from the same frames until we had 650 bees to form 420 a colony, which took typically 2-3 days. To achieve relatively normal conditions for 421 typical honey bee behavior, we supplemented workers from an unselected colony 422 (control bees), who were not marked. For colony set up, see Supplementary Table 1.

423 Bees were then placed into 4 different treatment colonies consisting of $\sim 1300$ bees:

424 high plus controls, low plus controls, 50/50 mixed high/low plus controls, and only 425 control colony. Bees were provided a honeycomb and remained inside for 5 days before being placed for field experimentation. We then placed nucleus colonies into outdoor

427 flight cages $(3.05 \mathrm{~m} \times 12 \mathrm{~m})$ and replaced the honeycomb frame with an empty frame to 428 induce foraging the night before the experiment. Water was provided as needed. We ran high, low, mixed, and control colonies concurrently in 4 different tents.

432 in the same location all week and became the 'familiar' feeder (Figure 2). We then 433 placed one novel feeder in different locations each day (Day 2 (X), Day 3 (Y), and Day 4

$434(Z)$ ). Feeders had unique colors and unique odors and remained consistent throughout 435 the experiment (Supplementary Table 2). 
To evaluate round dance behavior of each of the selected lines, we created 6 50/50 mixed colonies as detailed above. To induce foraging behavior, we placed the colonies in a climate controlled indoor room for 10 days to allow bees to age which

442 increases foraging behavior. After 10 days, we then placed all bees from each colony

443 into a two-frame observation hive with glass walls. All comb surfaces were visible. We

444 video recorded round dance behavior using a Panasonic HC-WXF991K, starting the

445 recording 15 minutes before feeders were placed in the flight cage. For distances from

446 the colony entrance, see figure 4A. We followed the same feeder placement pattern

447 across 4 days, from Monday to Thursday, in Figure 4A. Round dance data was then

448 extracted visually from watching videos. We recorded the LI line of the dancer according

449 to the color marking on her thorax color, the feeder she visited according to the color

450 mark on her abdomen (which also distinguished her as having visited a feeder),

451 duration of the round dance, the LI line of the round dance followers, and the number of

452 turns in a dance during the first $20 \mathrm{~s}$ of the dance, or less if the dance ended before $20 \mathrm{~s}$

453 elapsed. As the feeders were less than $12 \mathrm{~m}$ away from the colony, bees performed

454 round dances which lack distinct 'runs' and often have incomplete turns. Video watchers

455 were blind to the thorax and abdomen color associations between LI line and feeder

456 visitation, respectively.

Data Analysis Methods

To test whether bees exhibited a similar LI score as their parents regardless of where they were housed after emergence, we used a generalized linear model. We used LI score as the response variable, which fit a log-linear distribution, so we used a

462 gaussian family with a log link. Our fixed predictor variables were the line from which the 463 bees originated (high or low) and the colony type that they were placed in after 464 emergence (either their natal colony or a control colony).

465 To evaluate the effect of colony composition on colony-level foraging behavior to 466 novel and familiar feeders, we performed a general linear model with a gaussian error 467 distribution on number of visits, with line and feeder as fixed predictor variables, as well 468 as the interaction between line and feeder. We performed a generalized linear model with a binomial error distribution with a logit link function on percent revisitation, as it 
470 was a proportion comparing the number of revisits divided by the total number of visits.

471 Line and feeder were fixed predictor variables, as well as the interaction between the

472 line and feeder.

473 To explore whether the selected LI line of a forager bee influenced which feeder

474 it visited while in the mixed colony, we used a general linear model with a gaussian

475 error distribution on number of visits, with year, selected line and feeder as a fixed

476 predictor variables, as well as the interactions between these three. We did find a

477 significant three-way interaction between year, selected line, and feeder, which we

478 present in Supplementary Table 6. Therefore, we treat years independently and

479 performed two different GLMs with selected line and feeder as our fixed predictor

480 variables, as the workers from the different years came from a new set of selected

481 queens and drones, colonies were in nucleus Langstroth hive boxes in 2017 but were

482 placed in observation colonies in 2018, as well as differences in weather.

To compare the round dance behavior among the selected lines, we examined

484 the effect of dancer selected line on the duration of the round dance, intensity of

485 dancing, number of turns by dancers, and number of followers of each dance using

486 generalized linear models. To analyze whether the duration of the round dance differed

487 across the learning lines, the duration of the round dance response variable fit a log-

488 normal distribution, so we used a generalized linear mixed model with a gaussian family

489 and a log link. The LI of the dancer was our fixed predictor variable. To evaluate which

490 lines attracted more dancers, we used a chi-square test to compare the proportion of

491 dances that attracted no followers across the lines. To evaluate whether there were

492 differences in the number of turns the dancers from each line performed, we used a

493 linear mixed model because the response variable - the number of turns per second,

494 was normally distributed. Finally, we used a negative binomial mixed regression model

495 using the package MASS(39) to understand how duration of a dance and the LI of the

496 dancer interacted to predict the number of followers. 159 dances out of 908 total dances

497 had no followers, requiring a zero-inflated model approach. We analyzed only bees that

498 had paint marks on their abdomens, ensuring that they had visited a feeder.

499 We used an Analysis of Deviance Wald chi-square test using the function Anova

500 in the MASS(39) package to further evaluate the overall effect of each fixed predictor 
501 variable and interaction. We used the Ime4 package(40) to perform these tests unless

502 otherwise noted. Post hoc tests were performed to determine the relationships between

503 the different levels of fixed predictor variables and their interactions using the package

504 emmeans(41). We use R(42) for analysis.

505

506 Data Availability: Data will be available on FigShare and code will be available on

507 Github upon publication. Data and code available upon request by reviewers.

Ethical Compliance: Honey bees (Apis mellifera) were used in this study. Queens

510 (reproductive females) and drones (males) were behaviorally selected using lab assays

511 to create selected lines of colonies. Worker honey bees (non-reproductive females)

512 were tested in the field. All colonies were kept with typical honey bee practices. There

513 was no ethics committee involved in approving the animal husbandry protocol.

515 Acknowledgements: We thank S. Ohrt, E Sezen, N Kulkarni, and A Phillips for help

516 with data collection. We thank C. Kwapich and D. Charbonneau for comments on drafts

517 of this manuscript. This grant was funded by NIH NIGMS R01GM113967 to BHS, JG, \&

518 NPW, and NIH NIGMS F32GM126728 to CNC.

520 Competing Interests: The authors declare no competing financial interests 


\section{REFERENCES}

1. D. J. Sumpter, Collective animal behavior (Princeton University Press, 2010).

2. S. Camazine, et al., Self-organization in biological systems (Princeton university press, 2003).

3. I. D. Couzin, J. Krause, "Self-Organization and Collective Behavior in Vertebrates" in Advances in the Study of Behavior, (Elsevier, 2003), pp. 1-75.

538 4. I. D. Couzin, Collective cognition in animal groups. Trends Cogn. Sci. 13, 36-43 (2009).

5. M. del Mar Delgado, et al., The importance of individual variation in the dynamics of animal

6. I. D. Couzin, et al., Democratic Consensus in Animal Groups. 334, 4 (2011).

7. A. Sih, M. Del Giudice, Linking behavioural syndromes and cognition: a behavioural ecology

8. R. Dukas, Cognitive ecology: the evolutionary ecology of information processing and decision perspective. Philos. Trans. R. Soc. B Biol. Sci. 367, 2762-2772 (2012).

9. C. Ten Cate, C. Rowe, Biases in signal evolution: learning makes a difference. Trends Ecol. Evol. 22,

10. R. Schachar, et al., Restraint and Cancellation: Multiple Inhibition Deficits in Attention Deficit 380-387 (2007). Hyperactivity Disorder. J. Abnorm. Child Psychol. 35, 229-238 (2007).

11. P. Dayan, S. Kakade, P. R. Montague, Learning and selective attention. Nat. Neurosci. 3, 1218-1223 (2000).

12. C. J. Mitchell, M. E. Le Pelley, Attention and associative learning: From brain to behaviour (Oxford University Press, USA, 2010).

13. K. Von Frisch, L. E. Chadwick, The dance language and orientation of bees (Belknap Press of Harvard University Press Cambridge, MA, 1967).

14. T. D. Seeley, A. S. Mikheyev, G. J. Pagano, Dancing bees tune both duration and rate of waggle-run production in relation to nectar-source profitability. J. Comp. Physiol. A 186, 813-819 (2000).

15. J. C. Biesmeijer, T. D. Seeley, The use of waggle dance information by honey bees throughout their foraging careers. Behav. Ecol. Sociobiol. 59, 133-142 (2005).

16. C. I. Abramson, M. E. Bitterman, Latent inhibition in honeybees. Anim. Learing Behav. 14, 184-189 (1986).

17. H. J. Ferguson, S. Cobey, B. H. Smith, Sensitivity to a change in reward is heritable in the honeybee, Apis mellifera. Anim. Behav. 61, 527-534 (2001). 
18. M. D. Mitchell, M. I. McCormick, M. C. O. Ferrari, D. P. Chivers, Friend or foe? The role of latent inhibition in predator and non-predator labelling by coral reef fishes. Anim. Cogn. 14, 707-714 (2011).

19. M. C. O. Ferrari, D. P. Chivers, Latent inhibition of predator recognition by embryonic amphibians. Biol. Lett. 5, 160-162 (2009).

20. R. E. Lubow, Latent inhibition. Psychol. Bull. 79, 398 (1973).

21. R. E. Lubow, Latent inhibition as a measure of learned inattention: some problems and solutions. Behav. Brain Res. 88, 75-83 (1997).

22. R. E. Lubow, Latent inhibition: Effects of frequency of nonreinforced pre-exposure of the CS. $J$. Comp. Physiol. Psychol. 60, 454-457 (1965).

23. S. B. C. Chandra, J. S. Hosler, B. H. Smith, Heritable variation for latent inhibition and its correlation with reversal learning in honeybees (Apis mellifera). J. Comp. Psychol. 114, 86-97 (2000).

24. C. N. Cook, et al., Individual differences in learning and biogenic amine levels influence the behavioural division between foraging honeybee scouts and recruits. J. Anim. Ecol. 88, 236-246 (2018).

25. C. Brandes, R. Menzel, Common mechanisms in proboscis extension conditioning and visual learning revealed by genetic selection in honeybees (Apis mellifera). J. Comp. Physiol. A 166, 545552 (1990).

26. M. C. O. Ferrari, D. P. Chivers, Learning about non-predators and safe places: the forgotten elements of risk assessment. Anim. Cogn. 14, 309-316 (2011).

27. J. S. Latshaw, B. H. Smith, Heritable variation in learning performance affects foraging preferences in the honey bee (Apis mellifera). Behav. Ecol. Sociobiol. 58, 200-207 (2005).

28. K. D. Waddington, Honey bee foraging profitability and round dance correlates. J. Comp. Physiol. 148, 297-301 (1982).

29. P. I. Pavlov, Conditioned reflexes: an investigation of the physiological activity of the cerebral cortex. Ann. Neurosci. 17, 136 (1927).

30. R. E. Lubow, I. Weiner, P. Schnur, "Conditioned Attention Theory" in The Psychology of Learning and Motivation: Advances in Research and Theory., (Academic Press, 1981), pp. 1-49.

31. S. J. Gershman, D. M. Blei, Y. Niv, Context, learning, and extinction. Psychol. Rev. 117, 197-209 (2010).

32. R. E. Lubow, Latent inhibition and conditioned attention theory (Cambridge University Press, 1989).

33. H. R. Mattila, K. M. Burke, T. D. Seeley, Genetic diversity within honeybee colonies increases signal production by waggle-dancing foragers. Proc. R. Soc. B Biol. Sci. 275, 809-816 (2008).

34. R. Dukas, Behavioural and ecological consequences of limited attention. Philos. Trans. R. Soc. Lond. B. Biol. Sci. 357, 1539-1547 (2002). 
35. T. T. Hills, P. M. Todd, D. Lazer, A. D. Redish, I. D. Couzin, Exploration versus exploitation in space, mind, and society. Trends Cogn. Sci. 19, 46-54 (2015).

36. S. W. Cobey, D. R. Tarpy, J. Woyke, Standard methods for instrumental insemination of Apis mellifera queens. J. Apic. Res. 52, 1-18 (2013).

37. J. R. Harbo, "Propagation and instrumental insemination" in Bee Breeding and Genetics, Rinderer T.E., (Academic Press, Inc, 1986), pp. 361-389.

38. Z. S. Liang, et al., Molecular Determinants of Scouting Behavior in Honey Bees. Science 335, 12251228 (2012).

607 39. B. Ripley, et al., Package 'mass.' Cran R (2013).

40. D. Bates, M. Maechler, B. Bolker, S. Walker, lme4: Linear mixed-effects models using Eigen and S4. R Package Version 1, 1-23 (2014).

610 41. R. Lenth, M. R. Lenth, Package 'Ismeans.' Am. Stat. 34, 216-221 (2018).

611 42. R. C. Team, R: A language and environment for statistical computing (2018). 
631 SUPPMEMENTARY MATERIALS - Individual Learning Phenotypes Drive Collective

632 Cognition

633 Chelsea N. Cook ${ }^{1^{*}}$, Natalie J. Lemanski ${ }^{2}$, Thiago Mosqueiro², Jürgen Gadau ${ }^{3}$, Cahit Ozturk ,

634 Noa Pinter-Wollman ${ }^{2 \dagger}$, Brian H. Smith ${ }^{1 \dagger}$

635

636 Affiliations

$637{ }^{1}$ School of Life Sciences, Arizona State University, Tempe, USA

$638{ }^{2}$ Department of Ecology and Evolutionary Biology, University of California Los Angeles, Los

639 Angeles, USA

$640{ }^{3}$ Institute for Evolution and Biodiversity, University of Münster, Münster, Germany

$641{ }^{\dagger}$ Co-Senior Authors

$642 \quad{ }^{*}$ Corresponding Author, cncook1@asu.edu

643

644

645

646

647

648

649

650

651

652

653

654

655 


\begin{tabular}{|c|c|c|c|c|c|}
\hline & & \multicolumn{4}{|c|}{ Colony Type } \\
\hline & & High & Low & Mix & Control \\
\hline \multirow{4}{*}{ 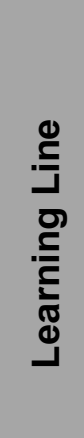 } & High LI & 650 & 0 & 325 & 0 \\
\hline & Low LI & 0 & 650 & 325 & 0 \\
\hline & Control & 650 & 650 & 650 & 1300 \\
\hline & Totals & 1300 & 1300 & 1300 & 1300 \\
\hline
\end{tabular}

657 Table S1: The number of honey bees in each experimental colony by genetic line. Each of

658 the 4 created colonies were set up in this way each week. We counted and marked the thorax

659 each bee from the learning lines, and counted but did not mark supplemental control bees.

\begin{tabular}{|c|c|c|c|}
\hline Day & $\begin{array}{c}\text { Feeder } \\
\text { Treatment }\end{array}$ & $\begin{array}{c}\text { Odor Added } \\
\text { to Feeder }\end{array}$ & $\begin{array}{c}\text { Color of } \\
\text { Feeder }\end{array}$ \\
\hline Day 1 & Familiar & Hexanol & Red \\
& & Hexanol + & Red + Blue \\
& Octanone & \\
\hline Day 2 & Familiar $+X$ & Hexanol + & Red + Pink \\
& & Geraniol & \\
\hline Day 3 & & & \\
& & & \\
& & &
\end{tabular}




\begin{tabular}{|l|l|l|l|} 
Day 4 & Familiar $+Z$ & Hexanol + & Red + Orange \\
& & Citranol & \\
& & \\
\hline
\end{tabular}

662 Table S2: The weekly routine of feeder characteristics and placement. Each feeder had 1M

663 sucrose solution. Color, odor, and location respectively varied by feeder. The treatment

664 sequence was the same each week.

665

666

667

668
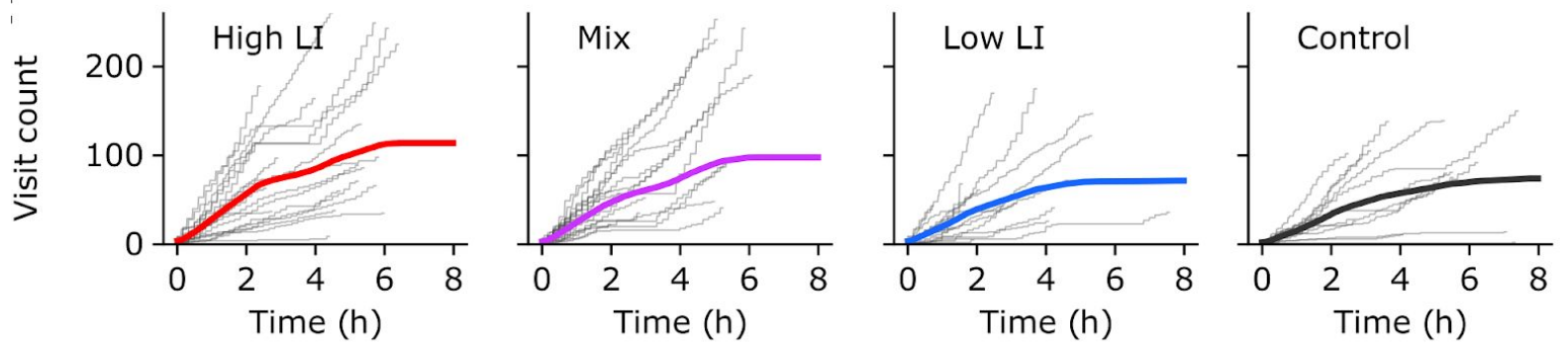

670

671 Figure S1: The cumulative visitation to all feeders over time, averaged across days. The

672 thick colored line is the average, and the gray stepwise lines are visitation on a single day by a

673 single colony. Colored lines are the same data shown in Figure 2B.

674

675

676

\begin{tabular}{|l|l|l|l|l|l|}
\hline contrast & estimate & SE & df & z.ratio & p.value \\
\hline control - high & 0.082 & 0.084 & Inf & 0.969 & 0.767 \\
\hline
\end{tabular}




\begin{tabular}{|l|l|l|l|l|l|}
\hline control - low & -0.065 & 0.099 & Inf & -0.659 & 0.912 \\
\hline control - mix & 0.293 & 0.088 & Inf & 3.336 & 0.005 \\
\hline high - low & -0.147 & 0.082 & Inf & -1.798 & 0.274 \\
\hline high - mix & 0.212 & 0.068 & Inf & 3.103 & 0.010 \\
\hline low - mix & 0.358 & 0.085 & Inf & 4.198 & 0.000 \\
\hline
\end{tabular}

678 Table S3: A table of the pairwise post hoc tests of how LI line predicts percent revisitation to all

679 feeders, referenced in figure $2 \mathrm{C}$.

\begin{tabular}{|l|l|l|l|l|l|}
\hline contrast & estimate & SE & df & z.ratio & p.value \\
\hline constant,control - novel,control & 0.163 & 0.080 & Inf & 2.031 & 0.461 \\
\hline constant,control - constant,high & -1.363 & 0.061 & Inf & -22.431 & 0.000 \\
\hline constant,control - novel,high & -0.249 & 0.072 & Inf & -3.437 & 0.014 \\
\hline constant,control - constant,low & -0.214 & 0.073 & Inf & -2.931 & 0.067 \\
\hline constant,control - novel,low & -0.298 & 0.072 & Inf & -4.162 & 0.001 \\
\hline constant,control - constant,mix & -1.467 & 0.060 & Inf & -24.393 & 0.000 \\
\hline constant,control - novel,mix & 0.219 & 0.081 & Inf & 2.701 & 0.122 \\
\hline novel,control - constant,high & -1.526 & 0.065 & Inf & -23.508 & 0.000 \\
\hline novel,control - novel,high & -0.411 & 0.076 & Inf & -5.421 & 0.000 \\
\hline novel,control - constant,low & -0.376 & 0.076 & Inf & -4.925 & 0.000 \\
\hline
\end{tabular}




\begin{tabular}{|c|c|c|c|c|c|}
\hline novel,control - novel,low & -0.460 & 0.075 & $\operatorname{lnf}$ & -6.129 & 0.000 \\
\hline novel,control - constant,mix & -1.630 & 0.064 & $\operatorname{lnf}$ & -25.338 & 0.000 \\
\hline novel,control - novel,mix & 0.057 & 0.084 & $\operatorname{lnf}$ & 0.675 & 0.998 \\
\hline constant,high - novel,high & 1.115 & 0.055 & $\operatorname{lnf}$ & 20.194 & 0.000 \\
\hline constant,high - constant,low & 1.150 & 0.056 & $\operatorname{lnf}$ & 20.555 & 0.000 \\
\hline constant,high - novel,low & 1.065 & 0.054 & $\operatorname{lnf}$ & 19.661 & 0.000 \\
\hline constant,high - constant,mix & -0.104 & 0.038 & $\operatorname{lnf}$ & -2.756 & 0.106 \\
\hline constant,high - novel,mix & 1.583 & 0.066 & $\operatorname{lnf}$ & 23.818 & 0.000 \\
\hline novel,high - constant,low & 0.035 & 0.068 & $\operatorname{lnf}$ & 0.512 & 1.000 \\
\hline novel,high - novel,low & -0.049 & 0.067 & $\operatorname{lnf}$ & -0.736 & 0.996 \\
\hline novel,high - constant,mix & -1.219 & 0.055 & Inf & -22.358 & 0.000 \\
\hline novel,high - novel,mix & 0.468 & 0.077 & $\operatorname{lnf}$ & 6.066 & 0.000 \\
\hline constant,low - novel,low & -0.084 & 0.068 & $\operatorname{lnf}$ & -1.248 & 0.918 \\
\hline constant,low - constant,mix & -1.254 & 0.055 & $\operatorname{lnf}$ & -22.690 & 0.000 \\
\hline constant,low - novel,mix & 0.433 & 0.078 & $\operatorname{lnf}$ & 5.574 & 0.000 \\
\hline novel,low - constant,mix & -1.170 & 0.053 & $\operatorname{lnf}$ & -21.864 & 0.000 \\
\hline novel,low - novel,mix & 0.517 & 0.076 & $\operatorname{lnf}$ & 6.767 & 0.000 \\
\hline constant,mix - novel,mix & 1.687 & 0.066 & Inf & 25.604 & 0.000 \\
\hline
\end{tabular}




\begin{tabular}{|c|c|c|c|c|c|}
\hline contrast & estimate & SE & df & z.ratio & p.value \\
\hline control,constant - high,constant & 0.251 & 0.127 & $\operatorname{lnf}$ & 1.975 & 0.499 \\
\hline control,constant - low,constant & -0.150 & 0.151 & $\operatorname{lnf}$ & -0.996 & 0.975 \\
\hline control,constant - mix,constant & 0.523 & 0.132 & $\operatorname{lnf}$ & 3.958 & 0.002 \\
\hline control,constant - control,novel & -0.972 & 0.147 & $\operatorname{lnf}$ & -6.635 & 0.000 \\
\hline control,constant - high,novel & -1.256 & 0.127 & $\operatorname{lnf}$ & -9.913 & 0.000 \\
\hline control,constant - low, novel & -0.881 & 0.141 & $\operatorname{lnf}$ & -6.231 & 0.000 \\
\hline control,constant - mix,novel & -1.394 & 0.135 & Inf & -10.311 & 0.000 \\
\hline high,constant - low,constant & -0.402 & 0.125 & Inf & -3.208 & 0.029 \\
\hline high,constant - mix,constant & 0.272 & 0.102 & $\operatorname{lnf}$ & 2.669 & 0.132 \\
\hline high,constant - control,novel & -1.223 & 0.120 & Inf & -10.209 & 0.000 \\
\hline high,constant - high,novel & -1.507 & 0.095 & $\operatorname{lnf}$ & -15.937 & 0.000 \\
\hline high,constant - low, novel & -1.133 & 0.114 & Inf & -9.972 & 0.000 \\
\hline high,constant - mix,novel & -1.645 & 0.106 & Inf & -15.566 & 0.000 \\
\hline low,constant - mix,constant & 0.673 & 0.130 & Inf & 5.174 & 0.000 \\
\hline low,constant - control,novel & -0.822 & 0.145 & Inf & -5.679 & 0.000 \\
\hline low,constant - high,novel & -1.105 & 0.125 & $\operatorname{lnf}$ & -8.873 & 0.000 \\
\hline low,constant - low,novel & -0.731 & 0.140 & $\operatorname{lnf}$ & -5.238 & 0.000 \\
\hline low,constant - mix,novel & -1.244 & 0.133 & Inf & -9.335 & 0.000 \\
\hline
\end{tabular}




\begin{tabular}{|l|l|l|l|l|l|}
\hline mix,constant - control,novel & -1.495 & 0.125 & Inf & -11.964 & 0.000 \\
\hline mix,constant - high,novel & -1.778 & 0.101 & Inf & -17.615 & 0.000 \\
\hline mix,constant - low,novel & -1.404 & 0.119 & Inf & -11.803 & 0.000 \\
\hline mix,constant - mix,novel & -1.917 & 0.111 & Inf & -17.197 & 0.000 \\
\hline control,novel - high,novel & -0.284 & 0.119 & Inf & -2.380 & 0.251 \\
\hline control,novel - low,novel & 0.091 & 0.135 & Inf & 0.673 & 0.998 \\
\hline control,novel - mix,novel & -0.422 & 0.128 & Inf & -3.291 & 0.022 \\
\hline high,novel - low,novel & 0.374 & 0.113 & Inf & 3.316 & 0.021 \\
\hline high,novel - mix,novel & -0.138 & 0.105 & Inf & -1.317 & 0.893 \\
\hline low,novel - mix,novel & -0.513 & 0.122 & Inf & -4.189 & 0.001 \\
\hline
\end{tabular}

686 Table S5: A table of the pairwise post hoc tests of how the Line*Feeder interaction predicts

687 percent revisitation, which corresponds to letters in figure $2 \mathrm{E}$.

\begin{tabular}{|l|l|l|l|}
\hline & LR Chisq & Df & Pr(>Chisq) \\
\hline BeeType & 100.073 & 2.000 & 0.000 \\
\hline Feeder & 2196.156 & 1.000 & 0.000 \\
\hline Year & 479.995 & 1.000 & 0.000 \\
\hline BeeType:Feeder & 47.718 & 2.000 & 0.000 \\
\hline BeeType:Year & 498.209 & 2.000 & 0.000 \\
\hline Feeder:Year & 61.341 & 1.000 & 0.000 \\
\hline BeeType:Feeder:Year & 31.492 & 2.000 & 0.000 \\
\hline
\end{tabular}


692 Table S6: Individual visitation by bee type differed across two experimental years. GLM

693 results showing the three-way interaction between year and the type of bee visiting a feeder

694 (Figure 3). There is likely a difference in year because of several reasons, including 1) Colonies

695 were selected from different queens from different breeders in 2017 and 2018 and climactic

696 conditions were different in 2017 compared to 2018 even though experiments were done in the

697 same time frame (June-July in 2017, June in 2018).

698

699

\begin{tabular}{|l|l|l|l|l|l|}
\hline contrast & estimate & SE & df & z.ratio & p.value \\
\hline control,familiar - high,familiar & -0.220 & 0.054 & Inf & -4.102 & 0.001 \\
\hline control,familiar - low,familiar & 0.624 & 0.068 & Inf & 9.210 & 0.000 \\
\hline control,familiar - control,novel & 1.387 & 0.076 & Inf & 18.320 & 0.000 \\
\hline control,familiar - high,novel & 1.425 & 0.077 & Inf & 18.564 & 0.000 \\
\hline control,familiar - low,novel & 1.986 & 0.095 & Inf & 20.794 & 0.000 \\
\hline high,familiar - low,familiar & 0.844 & 0.065 & Inf & 12.916 & 0.000 \\
\hline high,familiar - control,novel & 1.607 & 0.074 & Inf & 21.841 & 0.000 \\
\hline high,familiar - high,novel & 1.645 & 0.075 & Inf & 22.033 & 0.000 \\
\hline high,familiar - low,novel & 2.206 & 0.094 & Inf & 23.512 & 0.000 \\
\hline low,familiar - control,novel & 0.763 & 0.084 & Inf & 9.049 & 0.000 \\
\hline low,familiar - high,novel & 0.801 & 0.085 & Inf & 9.394 & 0.000 \\
\hline
\end{tabular}




\begin{tabular}{|l|l|l|l|l|l|}
\hline low,familiar - low,novel & 1.362 & 0.102 & Inf & 13.290 & 0.000 \\
\hline control,novel - high,novel & 0.038 & 0.092 & Inf & 0.413 & 0.998 \\
\hline control,novel - low,novel & 0.599 & 0.108 & Inf & 5.546 & 0.000 \\
\hline high,novel - low,novel & 0.561 & 0.109 & Inf & 5.159 & 0.000 \\
\hline
\end{tabular}

701 number of visits by each line in the mixed colonies in 2017 , which corresponds to letters in

702 figure $3 \mathrm{~A}$.

703

704

705

706

\begin{tabular}{|l|l|l|l|l|l|}
\hline contrast & estimate & SE & df & z.ratio & p.value \\
\hline control,familiar - high,familiar & 0.389 & 0.043 & Inf & 8.949 & 0.000 \\
\hline control,familiar - low,familiar & -0.227 & 0.037 & Inf & -6.117 & 0.000 \\
\hline control,familiar - control,novel & 0.567 & 0.041 & Inf & 13.904 & 0.000 \\
\hline control,familiar - high,novel & 1.451 & 0.054 & Inf & 26.775 & 0.000 \\
\hline control,familiar - low,novel & 0.827 & 0.044 & Inf & 18.827 & 0.000 \\
\hline high,familiar - low,familiar & -0.616 & 0.042 & Inf & -14.779 & 0.000 \\
\hline high,familiar - control,novel & 0.178 & 0.045 & Inf & 3.947 & 0.001 \\
\hline high,familiar - high,novel & 1.062 & 0.057 & Inf & 18.485 & 0.000 \\
\hline
\end{tabular}




\begin{tabular}{|l|l|l|l|l|l|}
\hline high,familiar - low,novel & 0.438 & 0.048 & Inf & 9.140 & 0.000 \\
\hline low,familiar - control,novel & 0.793 & 0.039 & Inf & 20.441 & 0.000 \\
\hline low,familiar - high,novel & 1.678 & 0.053 & Inf & 31.807 & 0.000 \\
\hline low,familiar - low,novel & 1.053 & 0.042 & Inf & 25.015 & 0.000 \\
\hline control,novel - high,novel & 0.884 & 0.055 & Inf & 15.959 & 0.000 \\
\hline control,novel - low,novel & 0.260 & 0.045 & Inf & 5.725 & 0.000 \\
\hline high,novel - low,novel & -0.625 & 0.058 & Inf & -10.810 & 0.000 \\
\hline
\end{tabular}

708 number of visits by each line in the mixed colonies in 2018 , which corresponds to letters in

709 figure 3B. 